\title{
DelosDLMS - the Integrated DELOS Digital Library Management System
}

\author{
Maristella Agosti ${ }^{1}$, Stefano Berretti ${ }^{2}$, Gert Brettlecker ${ }^{3}$, Alberto del Bimbo ${ }^{2}$, \\ Nicola Ferro ${ }^{1}$, Norbert Fuhr ${ }^{4}$, Daniel Keim ${ }^{5}$, Claus-Peter Klas ${ }^{4}$, Thomas \\ Lidy $^{6}$, Diego Milano ${ }^{3,8}$, Moira Norrie ${ }^{7}$, Paola Ranaldi ${ }^{3,8}$, Andreas Rauber ${ }^{6}$, \\ Hans-Jörg Schek ${ }^{8}$, Tobias Schreck ${ }^{5}$, Heiko Schuldt ${ }^{3}$, Beat Signer ${ }^{7}$, and \\ Michael Springmann ${ }^{3}$ \\ 1 Information Management Systems Research Group, University of Padua (IT) \\ 2 Visual Information Processing Laboratory, University of Florence (IT) \\ 3 Database and Information Systems Group, University of Basel (CH) \\ 4 Information Systems Group, University of Duisburg-Essen (DE) \\ 5 Databases, Data Mining and Visualization Group, University of Constance (DE) \\ 6 Information \& Software Engineering Group, Technical University of Vienna (AT) \\ 7 Institute for Information Systems, ETH Zürich (CH) \\ 8 Database and Information Systems Group, University of Constance (DE)
}

\begin{abstract}
DelosDLMS is a prototype of a next-generation Digital Library (DL) management system. It is realized by combining various specialized DL functionalities provided by partners of the DELOS network of excellence. Currently, DelosDLMS combines text and audio-visual searching, offers new information visualization and relevance feedback tools, provides novel interfaces, allows retrieved information to be annotated and processed, integrates and processes sensor data streams, and finally, from a systems engineering point of view, is easily configured and adapted while being reliable and scalable. The prototype is based on the OSIRIS/ISIS platform, a middleware environment developed by ETH Zürich and now being extended at the University of Basel.
\end{abstract}

Key words: Digital Library Management System, SOA

\section{Introduction}

The overall goal of the DelosDLMS is the implementation of a prototype of a next-generation Digital Library management system [1]. This system combines text and audio-visual searching, offers personalized browsing using new information visualization and relevance feedback tools, provides novel interfaces, allows retrieved information to be annotated and processed, integrates and processes sensor data streams, and finally, from a systems engineering point of view, is easily configured and adapted while being reliable and scalable.

Previous work in the DELOS network has mainly focused on improving Digital Libraries (DLs) by developing independent, powerful and highly sophisticated prototype systems in cooperation of few partners. In addition, a dedicated

Konstanzer Online-Publikations-System (KOPS)

URN: http://nbn-resolving.de/urn:nbn:de:bsz:352-opus-68631

URL: http://kops.ub.uni-konstanz.de/volltexte/2009/6863 
DELOS activity is the definition of a Reference Model for Digital Libraries [2]. The goal of the DELOS integration activities is twofold. First, it aims at integrating these prototype systems as building blocks into OSIRIS/ISIS, an existing middleware environment that was developed at ETH Zurich. The result of the integration - that is, the middleware infrastructure together with all the advanced DL functionality - constitutes the DelosDLMS prototype. Second, DelosDLMS will serve as a partial implementation of the DELOS Reference Model.

This paper reports on the integration of DL functionality which includes audio (TU Vienna) and 3D features (Univ. Florence), a new paper-based interface (iPaper from ETH Zürich), a SOM visualization of the feature space (Univ. Konstanz), and an annotation service (FAST, Univ. Padua) which have been added to DelosDLMS and the integration of DelosDLMS into the Daffodil interface.

The paper is organized as follows. Section 2 introduces the ISIS/OSIRIS middleware which is the basis for DelosDLMS. Section 3 presents in detail the services and DL functionality that has been added. Section 4 concludes.

\section{OSIRIS/ISIS Middleware}

OSIRIS (Open Service Infrastructure for Reliable and Integrated process Support) is a platform that allows for combining different distributed services into processes [3]. The OSIRIS platform itself does not provide any application functionality but, by combining specialized application services, supports the definition and reliable execution of dedicated processes (also known as "programmingin-the-large"). When different specialized DL application services are made available to the OSIRIS platform, users can define and run powerful Digital Library processes by making use of these services. This is realized by a set of generic (application-independent) services that include the registration of services and processes, interfaces for application development, an engine for decentralized execution of processes, and services for load balancing. OSIRIS processes themselves are wrapped by a service interface. Therefore, a process can be invoked just like any other service (and used in other processes as well).

OSIRIS distinguishes between system services and application services. System services are used internally for coordinating the execution of processes in a distributed way, without relying on a central execution engine/scheduler. For application services, OSIRIS further distinguishes between loosely coupled and tightly coupled services. Usually, a part of the distributed OSIRIS middleware (called OSIRIS layer) runs on each host providing application services. This is the case for tightly coupled services. Loosely coupled application services are those that have to be called remotely, without a local ORISIS layer available. The integration/invocation is done via WSDL for service description and SOAP for invocation. The system architecture of OSIRIS is depicted in Figure 1. Another focus of OSIRIS is scalability of process execution. OSIRIS' decentralized peer-to-peer approach for process execution, which is realized by sophisticated replication mechanisms for control flow dependencies, avoids any single point of failure during process execution and provides a high degree of scalability. Peer- 


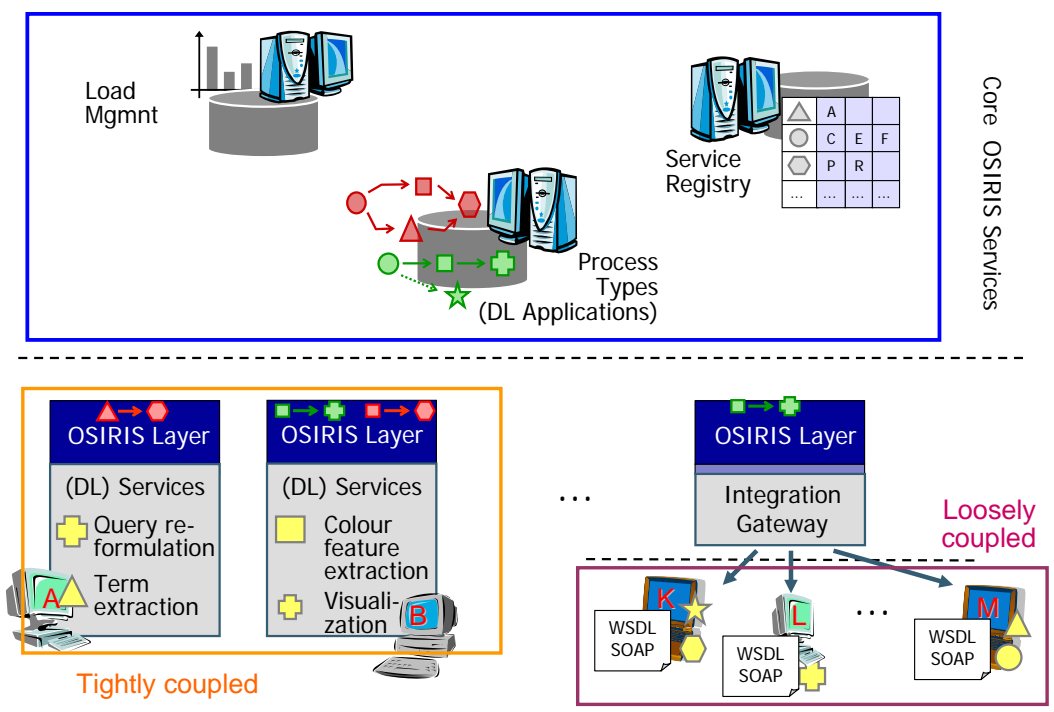

Fig. 1. The OSIRIS Middleware at a Glance

to-peer process execution also incorporates sophisticated load balancing in order to distribute process load among available, suitable peers.

Finally, OSIRIS is equipped with the O'GRAPE (Osiris GRAphical Process Editor) user interface for process definition. In addition, O'GRAPE supports the integration of existing application services via existing Web service standards (SOAP and WSDL).

The core of OSIRIS has been developed at ETH Zürich and is currently being extended at the University of Basel. There are two versions of OSIRIS: one is implemented in $\mathrm{C}++$ and runs on Microsoft platforms, the other version is implemented in Java.

ISIS (Interactive SImilarity Search) is a prototype application for information retrieval in multimedia collections [4]. It supports content-based retrieval of images, audio and video content, and the combination of any of these media types with text retrieval. Basically, ISIS consists of a set of pre-defined processes and several application services (like feature extraction, index management, index access, relevance feedback, etc.) that have been developed on the basis of the OSIRIS middleware. ISIS includes a sophisticated index structure (VA-file) for similarity search, which is particularly well suited for high-dimensional vector spaces. It also provides basic support for relevance feedback and visualization.

With the DelosDLMS, existing ISIS services are significantly enriched by other specialized DL services that have been developed within the DELOS network. This is achieved by integrating these services into the OSIRIS infrastructure, thereby combining them with other ISIS and non-ISIS services into advanced, process-based DL applications. 


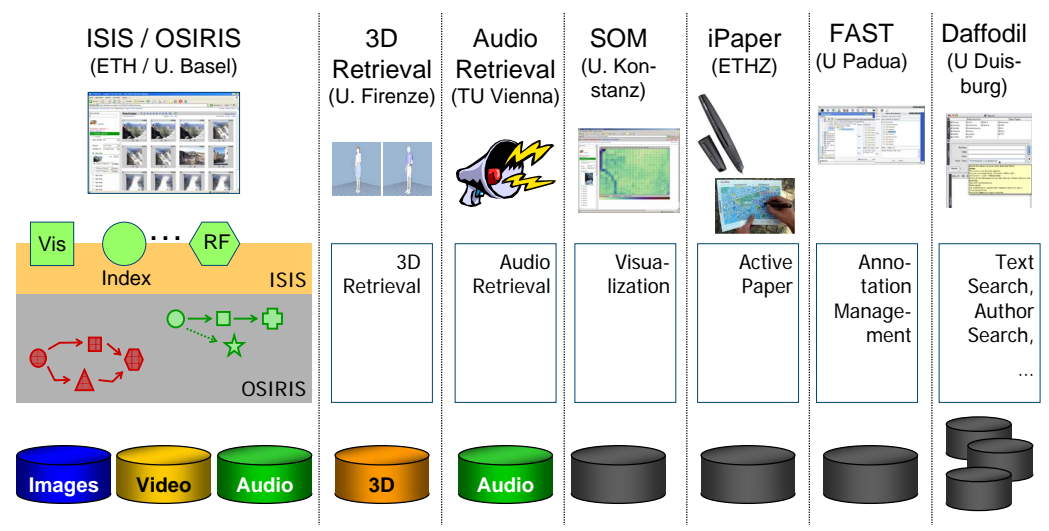

Fig. 2. Overview of Systems which have been integrated into DelosDLMS

\section{Integration Activities}

The starting point for the DelosDLMS integration activities is illustrated in Figure 2. In what follows, all these components are presented in more detail.

\subsection{Content-Based 3D Retrieval}

Histograms of surface curvature have been used to support global description and retrieval of $3 \mathrm{D}$ objects. However, since histograms do not include any spatial information, they are liable to false positives. To overcome such problems, we used curvature correlograms as a model for representation and retrieval of 3D objects $[5,6]$. Correlograms have been previously used with success for retrieval of images based on colour content [7]. In particular, with respect to description based on histograms of local features, correlograms enable also encoding of information about the relative position of local features. Correlograms are used to encode information about curvature values and their localization on the object surface. For this peculiarity, description of 3D objects based on correlograms of curvature proves to be effective for the purpose of content based retrieval of $3 \mathrm{D}$ objects. In order to compute curvature correlograms of a $3 \mathrm{D}$ object, the principal curvatures are computed for every vertices of the mesh. Values of the mean curvature are then derived from principal curvature, and quantized into N classes of discrete values. For this purpose, the mean curvature value is processed through a stair-step function so that many neighbouring values are mapped to one output value.

The proposed retrieval approach has been applied to the models included in the Princeton Shape Benchmark database [8]. This archive includes 1814 models categorized in a hierarchy of classes. In DelosDLMS, the curvature correlograms of the Princeton 3D-collection have been extracted and indexed with ISIS' VA- 

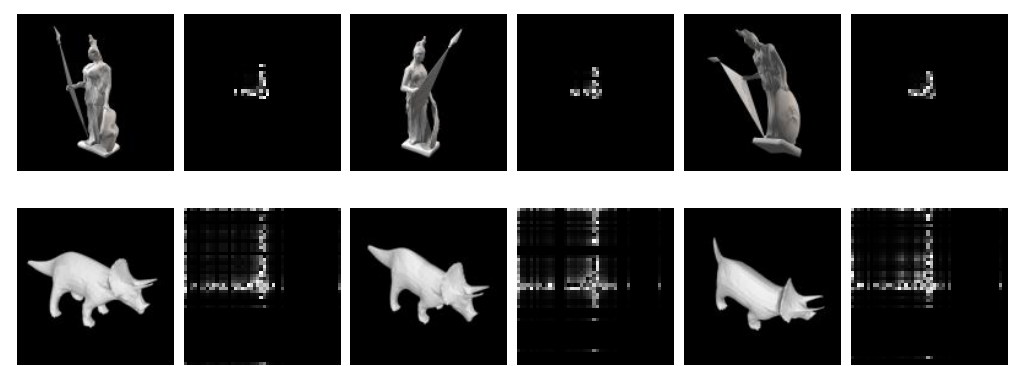

Fig. 3. Correlograms of 3 Models from 2 distinct Categories (Statue \& Dinosaur)

file and a Web service offering online feature extraction by Delos DLMS has been developed.

\section{$3.2 \quad$ Audio Retrieval}

TU Vienna has developed three different feature sets for content-based music retrieval. These feature sets for musical content description - Rhythm Patterns, Statistical Spectrum Descriptors and Rhythm Histograms - are integrated into DelosDLMS. The feature extraction algorithm is as follows:

In a pre-processing step the audio signal is converted to a mono signal and segmented into chunks of approximately 6 seconds. Typically, the first and last one or two segments are skipped and from the remaining segments every third one is processed.

For each segment the spectrogram of the audio is computed using the short time Fast Fourier Transform (STFT). The Bark scale, a perceptual scale which groups frequencies to critical bands according to perceptive pitch regions, is applied to the spectrogram, aggregating it to 24 frequency bands.

From this representation of perceived loudness statistical measures (mean, median, variance, skewness, kurtosis, min and max) are computed per critical band, in order to describe fluctuations within the bands extensively. The se result is a Statistical Spectrum Descriptor.

In a further step, the varying energy on the critical bands of the Bark scale Sonogram is regarded as a modulation of the amplitude over time. Using a Fourier Transform, the spectrum of this modulation signal is retrieved. The result is a time-invariant signal that contains magnitudes of modulation per modulation frequency per critical band. This matrix represents a Rhythm Pattern, indicating occurrence of rhythm as vertical bars, but also describing smaller fluctuations on all frequency bands of the human auditory range. Subsequent to the Fourier Transform, modulation amplitudes are weighted according to a function of human sensation of modulation frequency, accentuating values around $4 \mathrm{~Hz}$. The application of a gradient filter and Gaussian smoothing potentially improves similarity of Rhythm Patterns which is useful in classification and retrieval tasks.

A Rhythm Histogram is constructed by aggregating the critical bands of the Rhythm Pattern (before weighting and smoothing), resulting in a histogram of 

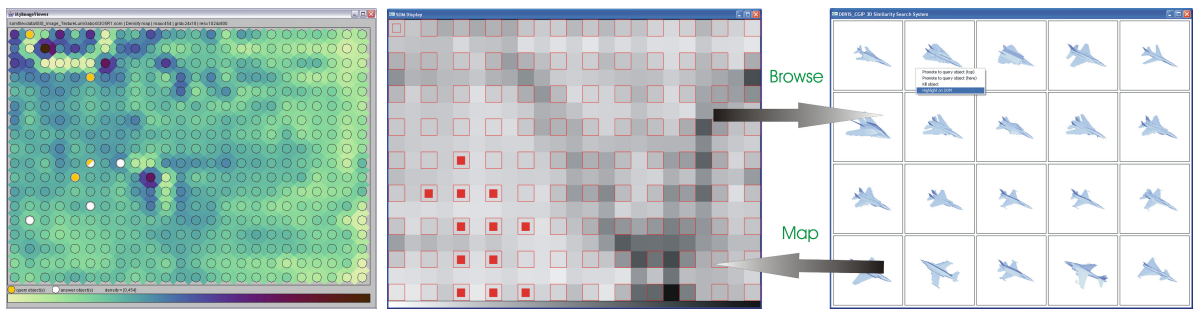

Fig. 4. Left: SOM for DelosDLMS Database Content; Right: SOM-Based Database Browsing and Exploration Concept

rhythmic energy for 60 modulation frequencies. The feature vectors are computed for a piece of audio by taking the median of the descriptors of its segments.

\subsection{SOM-Based Retrieval Support}

A standard method to visualize the result set of a user query in a DL is to display a sorted list of answer objects. Visualizing multiple object sets at once, as well as their structure and interrelationships between each other, possibly in different feature spaces, is feasible with projections from high-dimensional feature space to low-dimensional (2D) display space. Among other algorithms such as Principal Component Analysis and Multidimensional Scaling, the Self-Organizing Map (SOM) algorithm is a projection and vector quantization algorithm with interesting theoretical and practical properties, which is also highly suited for visualization. Previous work done at the University of Constance has focused on the application of the SOM algorithm for effective retrieval and visual analysis in multimedia databases. For DELOS, this work was leveraged in computing SOM projections for an image database described in different feature spaces. A web service was developed, which, based on the offline calculated maps, is able to render two basic SOM views. Query and result objects are marked on the map. The overall goal of this visualization is to support the user in analyzing and comparing the relationships between query and result sets, possibly under different feature representations. Furthermore, the visualization allows the user to effectively explore the contents and structure of a previously unknown data repository (visual analysis and scatter browsing).

\subsection{Daffodil}

The DL system Daffodil is a front-end system for Digital Libraries, targeted at strategic support of users during the information search process. For searching, exploring, and managing DL objects it provides user-customisable information seeking patterns over a federation of heterogeneous DLs. Searching with Daffodil makes a broad range of information sources easily accessible and enables quick access to a rich information space. Daffodil already provides a broad range of 
tools to help the user in query formulation, e.g. a thesaurus, a spell-checker, a classification browser, and a related-term service. For the user interface design, Daffodil implements a tool-based design paradigm, where objects resulting from the interaction with one tool can be dragged to other tools for further processing. Based on this metaphor, Daffodil implements the user-oriented integration of DL tools in the DelosDLMS, thus complementing the system-oriented integration performed by the OSIRIS middleware.

As a first reference implementation, the Daffodil system was extended by an image search tool connecting to the ISIS system described above. For this purpose, the Daffodil system was extended by two special functions: (i.) Query by Example: Given any picture link from the Web, similar pictures are searched. The similarity feature can be chosen, e.g. colour histograms. A set of answers pictures is returned. The pictures can then be used for further searching. (ii.) Query by Keyword: Given a specific keyword, the system is able to search for pictures, which are indexed with this keyword.

A feedback list is implemented in both functions. The user is able to specify relevant or non-relevant pictures in order to refine the answer set of searched pictures. The communication between OSIRIS and Daffodil is handled by HTTP and based on the processes given by the ISIS picture search. Through the integration within the Daffodil system, users can also apply other tools on queries and retrieved images, like maintaining a search history, storing queries and images within their personal libraries, sharing them with other users or adding annotations (see Section 3.6).

\section{5 iPaper}

Information managed by the DelosDLMS can be accessed by a variety of digital user interfaces. The goal of the iPaper extension for the DelosDLMS was to enhance existing paper documents with functionality offered by DelosDLMS thereby bridging the gap between paper documents and services offered by Digital Library management systems.

We developed an interactive printed museum guide as a physical user interface to the DelosDLMS. The system is based on a Bluetooth-enabled digital pen that can track user interactions within paper documents or any other surface covered with a special printed Anoto pattern consisting of tiny, almost invisible dots. Active paper areas can be defined within paper documents and linked to digital information or services. In the case of the interactive museum guide shown in Figure 5, existing printed text components and images, as well as some new interface components in the form of blue "paper buttons" have been associated with specific ISIS queries.

A first query that can be formulated based on the paper interface is retrieving images based on a set of given keywords. A user can either select specific underlined words within the text, which is similar to selecting hyperlinks on the Web, or they can enter specific words in a special keyword input area. Any keywords entered in the keyword area are transformed to a text string by an Intelligent Character Recognition (ICR) process. As soon as the user selects the 'Search' 


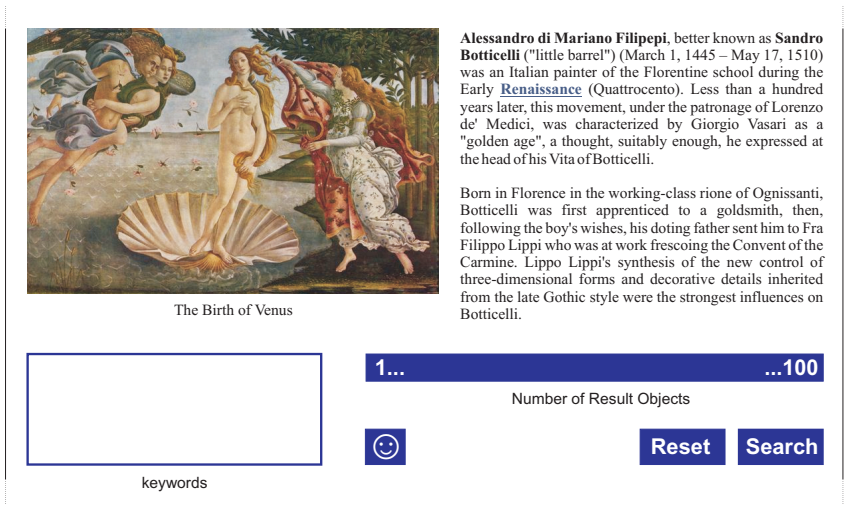

Fig. 5. Paper-based DelosDLMS Query Interface

button with the digital pen, a query consisting of the set of keywords is sent to ISIS and a list of images matching these keywords is returned. Another type of query is based on finding image similarities. By touching images in the museum guide with the digital pen, a user can add them to the set of images to be used in a similarity search. After selecting the 'Search' button, a query with the set of selected images as parameter is sent to ISIS and a list of best matching images is returned. Note that the keyword and image-based searches may be combined and queries containing keywords as well as images can be formulated. Finally, there are some paper buttons to configure specific query parameters such as the preferred size of the result set (from 1 up to 100 images).

The iPaper infrastructure $[9,10]$ used to implement the paper-based interface for DelosDLMS was developed by the Global Information Systems (GlobIS) research group at ETH Zürich. It is based on a general cross-media information management server (iServer) [11] enabling the integration of arbitrary digital and physical resources.

\subsection{FAST Annotation}

FAST [12] is a flexible system designed to support both various architectural paradigms and a wide range of different DLMSs. In order to achieve the desired flexibility: (i.) FAST is a stand-alone system, i.e., it is not part of any particular DLMS; (ii.) the core functionalities of the annotation service are separated from the functionalities needed to integrate it into different DLMSs. The flexibility of FAST and its independence from any particular DLMS are key features to provide users with a uniform way of interaction with annotation functionalities. In this way, users do not need to change their annotative practices only because they work with different DLMSs.

Within FAST, annotations are composite multimedia objects, which can annotate multiple part of a given digital object and can relate this annotated digital object to various other digital objects, if needed. Furthermore, once it has been 


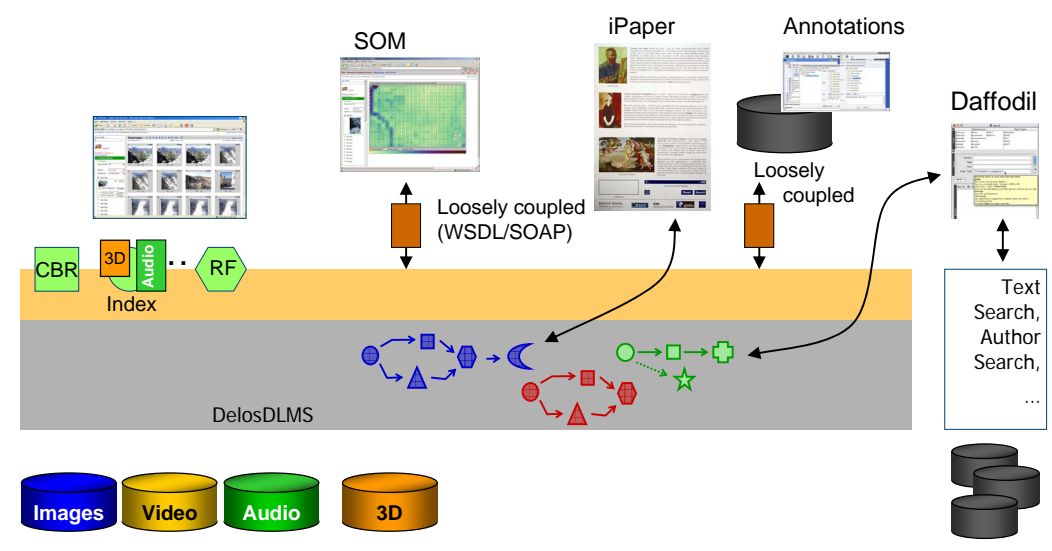

Fig. 6. Summary of the First Phase of DelosDLMS Service Integration

created, an annotation is considered as a first class digital object, so that it can be annotated too. In this way, users can create not only sets of annotations concerning a digital object, but also threads of annotations, i.e., annotations which reply to one another. These threads of annotations are the basis for actively involving users with the system and for enabling collaboration.

From a functional point of view, FAST offers annotation management functionalities, such as creation, access, and so on. Furthermore it supports collaboration among user by introducing scopes of annotation and groups of users: annotations can be private, shared or public; public; if an annotation is shared, different groups of users can share it with different permissions, e.g., one group can only read the annotation while another can also modify it. Finally, FAST offers advanced search functionalities based on annotations by exploiting annotations as a useful context in order to search and retrieve relevant documents for a user query $[13,14]$. The aim is to retrieve more documents that are relevant and to have them ranked in a way which is better than a system that does not makes use of annotations.

\subsection{Summary of Integration Activities}

All integration activities of the first phase are summarized in Figure 6. Audio and 3D features have been added to the VA-file index of DelosDLMS. The FAST annotation management and the SOM visualization are available as web services and have been loosely integrated into DelosDLMS. For this, OSIRIS processes have been extended accordingly in order to make use of these new services. The iPaper front-end creates a query which is sent and executed in DelosDLMS. Similarly, the Daffodil client generates a query which is sent to DelosDLMS. 


\section{Summary and Outlook}

The DelosDLMS prototype development will first continue with a tight integration of services already coupled in a loosely way. Second, functionality (espacially for feature extraction) which has been integrated off-line will be made online as dedicated Web service. It will then be possible to create OSIRIS processes that allow for the upload and indexing of new objects and/or for searching with objects that are not yet indexed. Third, new functionality will be added. This includes personalisation, term extraction, query support for XML documents, semantic video services, video annotation, and video retrieval, $\mathrm{P} 2 \mathrm{P}$ search, natural language and speech interfaces and further visualizations.

\section{References}

1. Schek, H.J., Schuldt, H.: DelosDLMS - Infrastructure for the Next Generation of Digital Library Management Systems. ERCIM, Special Issue on European Digital Library, (66) (July 2006)

2. Candela, L., Castelli, D., Pagano, P., Thanos, C., Ioannidis, Y., Koutrika, G., Ross, S., Schek, H.J., Schuldt, H.: Setting the Foundations of Digital Libraries: The DELOS Manifesto. D-Lib Magazine 13 (2007)

3. Schuler, C., Türker, C., Schek, H.J., Weber, R., H., S.: Scalable Peer-to-Peer Process Management. International Journal of Business Process Integration and Management (IJBPIM) 1(2) (2006) 129-142

4. Mlivoncic, M., Schuler, C., Türker, C.: Hyperdatabase Infrastructure for Management and Search of Multimedia Collections. In: Proc. DELOS Workshop. (2004)

5. Antini, G., Berretti, S., Bimbo, A.D., Pala, P.: Curvature Correlograms for Content Based Retrieval of 3D Objects. In: Proc. ICIAP'2005, Cagliari, Italy (2005)

6. Antini, G., Berretti, S., Bimbo, A.D., Pala, P.: Retrieval of 3D Objects using Curvature Correlograms. In: Proc. ICME'05, Amsterdam, The Netherlands (2005)

7. Huang, J., Kumar, R., Mitra, M., Zhu, W.J., R., Z.: Spatial Color Indexing and Application. International Journal of Computer Vision 35 (1999) 245-268

8. Shilane, P., Kazhdan, M., Min, P., Funkhouser, T.: The Princeton Shape Benchmark (2004) Shape Modeling International, Genova, Italy.

9. Norrie, M., Signer, B., Weibel, N.: General Framework for the Rapid Development of Interactive Paper Applications. In: Proc. CoPADD'2006, Banff, Canada (2006)

10. Norrie, M.C., Palinginis, A., Signer, B.: Content publishing framework for interactive paper documents. In: ACM Symp. on Document Engineering. (2005)

11. Norrie, M.C., Signer, B.: Information Server for highly-connected cross-media Publishing. Inf. Syst. 30(7) (2005) 526-542

12. Agosti, M., Ferro, N.: A System Architecture as a Support to a Flexible Annotation Service. In: Proc. of the 6th Thematic DELOS Workshop. (2005) 147-166

13. Agosti, M., Ferro, N.: Annotations as Context for Searching Documents. In: Proc. CoLIS'2005, Glasgow, UK (2005)

14. Agosti, M., Ferro, N.: Search Strategies for Finding Annotations and Annotated Documents: The FAST Service. In: Proc. FQAS'2006, Milan, Italy (2006) 